Pacific Journal of Mathematics

THE AUTOMORPHISM GROUPS OF SPACES AND 


\title{
THE AUTOMORPHISM GROUPS OF SPACES AND FIBRATIONS
}

\author{
SARA HURVITZ
}

This paper deals with the automorphism group of fibrations $f: X \rightarrow Y$, where $X$ and $Y$ are simply connected $C W$ complexes with either a finite number of homology groups or homotopy groups. It is proved that the automorphism groups of such fibrations are finitely presented, and that in case $X$ and $Y$ are $H_{0}$-spaces the image of the obvious map $\operatorname{Aut}(f) \rightarrow \operatorname{Aut}\left(H^{*}(f, Z)\right)$ has finite index in $\operatorname{Aut}\left(H^{*}(f, Z)\right)$. It is also proved that in case that $Y$ belongs to the genus of $X, \operatorname{Ker}\left(\right.$ Aut $\left.X \rightarrow \operatorname{Aut} X_{p}\right)$ is isomorphic to $\operatorname{Ker}($ Aut $Y \rightarrow$ Aut $\left.Y_{p}\right)\left(()_{p}\right.$-localization of $\left.p\right)$.

Introduction. Let $X, Y$ be spaces and let $f: X \rightarrow Y$ be a fibration. This work concerns the group Aut $X$ of homotopy classes of self equivalences of $X$ as well as the group $\operatorname{Aut}(f)$ of homotopy classes of pairs $(h, k) \in$ Aut $X \times$ Aut $Y$ which satisfy $f h \sim k f$. Throughout this paper all spaces considered are of the homotopy type of nilpotent $C W$-complexes of finite type, and all, except those which appear in Chapter four, are of the homotopy type of simply connected $C W$-complexes, which are either finite dimensional or with a finite number of homotopy groups.

We use the notations of Wilkerson [8]. We recall that a space $X$ is called an $H_{0}$-space if $H^{*}(X, Q)$ is an exterior algebra on odd dimensional generators, that the genus of $X$ is the set $G(X)$ of homotopy types of spaces $Y$ with $Y_{p} \approx X_{p}$ for every prime $p$, and that the ele. ments $\left[f^{\prime}\right]$ of the genus of a fibration $f: X \rightarrow Y$ are equivalence classes of homotopy classes $f^{\prime}$ which satisfy: For every prime $p$ there exist homotopy equivalences $h_{p}: X_{p}^{\prime} \rightarrow X_{p}, k_{p}: Y_{p}^{\prime} \rightarrow Y_{p}$ satisfying $f_{p} h_{p} \sim k_{p} f_{p}^{\prime}$.

Concerning Aut $X$ and $\operatorname{Aut}(f)$ we are interested in the following questions:

(a) Is the group $\operatorname{Aut}(f)$ finitely presented? i.e., can Theorem $B$ in Wilkerson [8] be generalized to Aut $(f)$ ?

(b) What is the relation between:

(1) Aut $X$ and Aut $H^{*}(X, Z)$ where $X$ is an $H_{0}$-space.

(2) $\operatorname{Aut}(f)$ and Aut $H^{*}(f, Z)$ where $f$ is an $H_{0}$-fibration, i.e., $f$ is a fibration between $H_{0}$-spaces.

(3) Aut $X$ and Aut $X^{\prime}$ where $X^{\prime}$ belongs to the genus of $X$.

(4) $\operatorname{Aut}(f)$ and $\operatorname{Aut}\left(f^{\prime}\right)$ where $f^{\prime}$ belongs to the genus of $f$. The answer to question (a) is given by:

MaIN Theorem. Let $X, Y$ be simply connected $C W$-complexes 
and let $F \rightarrow X \stackrel{f}{\rightarrow} Y$ be a fibration. Then:

(a) $\operatorname{Aut}(f)$ is commensurable with an arithmetic subgroup of $\operatorname{Aut}\left(f_{0}\right)$, where $f_{0}: X_{0} \rightarrow Y_{0}$ is the rationalization of $f$.

(b) $\operatorname{Aut}(f)$ is finitely presented, and

(c) $\operatorname{Aut}(f)$ has only a finite number of finite subgroup up to conjugation.

One of the results of this theorem is:

CoROLlaRY 2.8. Let $X$ be a simply connected finite $C W$-complex and let $G \subseteq$ Aut $X$ be a finitely generated subgroup. If $H_{*}(X, Z)$ is torsion free then the centralizer of $G$ is finitely presented.

Concerning question (b) we obtain the following interesting results:

Proposition 3.2. Let $X, Y$ be $H_{0}$-spaces and let $f: X \rightarrow Y$ be a fibration. Then:

(a) The map $[Y, X] \rightarrow \operatorname{Hom}\left(H_{*}(Y, Z), H_{*}(X, Z)\right.$ is finite to one.

(b) $\operatorname{Im}\left(\right.$ Aut $X \rightarrow$ Aut $H^{*}(X, Z)$ is a subgroup of finite index.

(c) The kernel of the obvious map Aut $(f) \rightarrow$ Aut $H^{*}(f, Z)$ is finite and its image is a subgroup of finite index in Aut $H^{*}(f, Z)$.

(d) For any pair $(h, k) \in$ Aut $H^{*}(f, Z)$ there exists a pair $(\widetilde{h}, \widetilde{k}) \epsilon$ Aut $(f)$ and an integer $m$, so that $H^{*}(h, Z)=h^{m}$ and $H^{*}(k, Z)=k^{m}$.

Proposition 4.6. Let $X$ be an $H_{0}$-space either with a finite number of homology groups or with a finite number of homotopy groups. If $H^{*}(X, Z)$ is torsion free then $\operatorname{Ker}\left(\right.$ Aut $X \rightarrow$ Aut $\left.X_{q}\right) q \in P$ $(P$ - the set of primes) is a direct product of finite p-groups, $p \neq q$.

Proposition 4.7. Let $X, Y$ be nilpotent spaces with a finite number of homology groups and let $f: X \rightarrow Y$ be a fibration. Then for every prime $p$ and for every fibration $f^{\prime}: X^{\prime} \rightarrow Y^{\prime}$, which belongs to the genus of $f, \operatorname{Ker}\left(\operatorname{Aut}(f) \rightarrow \operatorname{Aut}\left(f_{p}\right)\right)$ is isomorphic to $\operatorname{Ker}\left(\operatorname{Aut}\left(f^{\prime}\right) \rightarrow\right.$ $\left.\operatorname{Aut}\left(f_{p}^{\prime}\right)\right)$.

As a consequence of Propositions 3.6 and 4.7 we obtain:

Proposition 3.7. Let $X, \mu_{0}$ be an $H_{0}$-space. Suppose $H^{*}\left(\mu_{0}, Q\right)$ is primitively generated, then the number of equivalence classes of $H$-structure on $X$ for which $H^{*}(\mu, Q)$ is equivalent to $H^{*}\left(\mu_{0}, Q\right)$ is finite.

CoROLlaRY 4.9. Let $X, Y$ be $H_{0}$-spaces either with a finite number 
of homology groups or with a finite number of homotopy groups and let $f: X \rightarrow Y$ be a fibration. Then for every fibration $f^{\prime}$ which belongs to the genus of $f, \operatorname{Ker}\left(\operatorname{Aut}(f) \rightarrow\right.$ Aut $\left.H_{*}(f, Z)\right)$ is isomorphic to $\operatorname{Ker}\left(\operatorname{Aut}\left(f^{\prime}\right) \rightarrow\right.$ Aut $\left.H_{*}\left(f^{\prime}, Z\right)\right)$.

Proposition 4.10. Let $f$ and $f^{\prime}$ be as in Corollary 4.9. If Aut $(f)$ is finite, then $\operatorname{Aut}(f)$ is isomorphic to $\operatorname{Aut}\left(f^{\prime}\right)$.

The paper is organized as follows:

In section one the relation between automorphism groups and rational equivalence is studied. The main result is proved in section two. In section three, the special properties of $H_{0}$-spaces and the results of section one are used to draw conclusions on the automorphism groups of $H_{0}$-spaces and fibrations. In the last section, section four, the relation between automorphism groups and genus is studied.

I am indebted to E. Dror and A. Zabordsky for encouragement and for several fruitful conversations.

\section{Automorphism groups and rational equivalence.}

LEMMA 1.1. Let $X, Y, X^{\prime}, Y^{\prime}$ be simply connected finite type $C W$-complexes, $f: X \rightarrow Y, f^{\prime}: X^{\prime} \rightarrow Y^{\prime}$ be fibrations and $F$ and $F^{\prime}$ be simple $C W$-complexes with $\pi_{*} F$ and $\pi_{*} F^{\prime \prime}$ finite dimensional and finite. Define $S$ to be the set of homotopy classes of pairs $(\varphi, \psi)$ satisfying:

(a) $\varphi: X \rightarrow X^{\prime}$ and $\psi: Y \rightarrow Y^{\prime}$ are maps with homotopy theoretic fibers $F$ and $F^{\prime}$, respectively.

(b) $f^{\prime} \varphi \sim \psi f$.

Then $\operatorname{Aut}(f)$ acts on $S$ and $S / \operatorname{Aut}(f)$ is a finite set.

Proof. Let $M$ be the set of triples $(\varphi, \psi, \widetilde{f})$, where $F \rightarrow \widetilde{X} \stackrel{\phi}{\rightarrow} X^{\prime}$ and $F^{\prime} \rightarrow \widetilde{Y} \stackrel{\varphi}{\rightarrow} Y^{\prime}$ are fibrations, $\tilde{f}: \tilde{X} \rightarrow \widetilde{Y}$ a map and $\psi \tilde{f} \sim f^{\prime} \varphi$. Define an equivalence relation on $M$ by: $(\varphi, \psi, \widetilde{f}) \sim\left(\varphi^{\prime}, \psi^{\prime}, \widetilde{f}^{\prime}\right)$ $\left(\tilde{f}^{\prime}: \tilde{X}^{\prime} \rightarrow \widetilde{Y}^{\prime}\right)$ if and only if there exist homotopy equivalences $\alpha: \widetilde{X} \rightarrow$ $\widetilde{X}^{\prime}, \beta: \widetilde{Y} \rightarrow \widetilde{Y}^{\prime}$ so that the following diagram homotopy commutes. For any pair $(\varphi, \psi) \in S$ there is a factorization of $\varphi$ and $\psi$ as $X \stackrel{i}{\rightarrow}$ $X_{\varphi} \stackrel{\tilde{\varphi}}{\rightarrow} X^{\prime}, Y^{j} \rightarrow Y_{\psi} \stackrel{\tilde{\psi}}{\rightarrow} Y^{\prime}$, where $i$ and $j$ are homotopy equivalences, $\widetilde{\varphi}$ and $\widetilde{\psi}$ are fibrations and $\widetilde{\varphi} i \sim \varphi, \widetilde{\psi} i \sim \psi$. Obviously $f^{\prime} \widetilde{\varphi} \sim \widetilde{\psi}\left(j f i^{-1}\right)$ $\left(i^{-1}=\right.$ the homotopy inverse of $\left.i\right)$ and therefore the triple $\left(\widetilde{\varphi}, \tilde{\psi}, j f i^{-1}\right) \epsilon$ $M$. Changing $(\varphi, \psi)$ within a homotopy class does not vary the equivalence class of the triple $\left(\widetilde{\varphi}, \widetilde{\psi}, j f i^{-1}\right)$. Hence $S \rightarrow M$ is well 


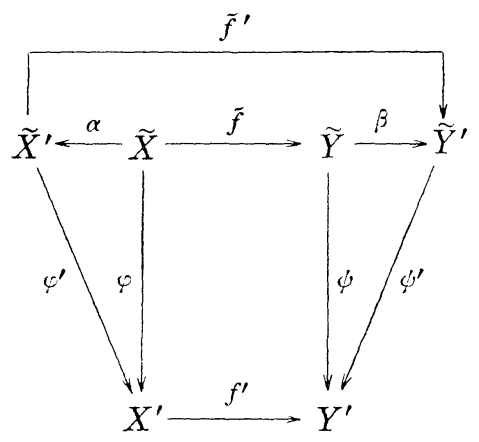

defined.

Suppose $(\varphi, \psi),\left(\varphi^{\prime}, \psi\right) \in S$ and there exists a pair $(\alpha, \beta) \in \operatorname{Aut}(f)$ so that $\varphi \alpha \sim \varphi^{\prime}$ and $\psi \beta \sim \psi^{\prime}$, then the triples $\left(\widetilde{\varphi}, \widetilde{\psi}, j f i^{-1}\right)$ and $\left(\widetilde{\varphi}^{\prime}, \widetilde{\psi}^{\prime}, j^{\prime} f i^{\prime-1}\right)$ are equivalent in $M$. Conversely, if the triples $\left(\widetilde{\varphi}, \widetilde{\psi}, j f i^{-1}\right)$ and $\left(\widetilde{\varphi}^{\prime}, \widetilde{\psi}^{\prime}, j^{\prime} f i^{\prime-1}\right)$ are equivalent in $M$ i.e., if there are homotopy equivalences $\alpha: X_{\varphi} \rightarrow X_{\varphi}, \beta: Y_{\psi} \rightarrow Y_{\psi}$ so that $\left(j^{\prime} f i^{\prime-1}\right) \alpha \sim$ $\beta\left(j f i^{-1}\right)$, then $\varphi\left(i^{-1} \alpha^{-1} i^{\prime}\right) \sim \varphi^{\prime}$ and $\psi\left(j^{-1} \beta j^{\prime}\right) \sim \psi^{\prime}$. Thus $S / \operatorname{Aut}(f) \rightarrow$ $M / \sim$ is well defined and monic. Therefore it is enough to prove that $M / \sim$ is finite. But by a standard Moore-Postnikov argument any element of $M$ can be obtained as a sequence of principal fibrations $\left(\varphi_{n}, \psi_{n}\right)$ with fibers $K\left(\pi_{n} X, n\right)$ and $K\left(\pi_{n} Y, n\right)$, so that $f_{n-1} \varphi_{n} \sim \psi_{n} f_{n}$. Hence it suffices to show that for each $n$ there is a finite number of equivalence classes of such fibrations, where the equivalence relation is defined as in $M$.

Suppose for the pair of $k$-invariants $\left(k, k^{\prime}\right) \in H^{n+1}\left(X_{n-1}, \pi_{n} X\right) \times$ $H^{n+1}\left(Y_{n-1}, \pi_{n} Y\right)$ there exists $f_{n}: X_{n} \rightarrow Y_{n}$ so that $f_{n-1} \varphi_{n} \sim \psi_{n} f_{n}$. Assume also that $\varphi_{n}^{\prime}: X_{n}^{\prime} \rightarrow X_{n-1}$ and $\psi_{n}^{\prime}: Y_{n}^{\prime} \rightarrow Y_{n-1}$ are fibers of $k$ and $k^{\prime}$, respectively, and there exists $f_{n}^{\prime}: X_{n}^{\prime} \rightarrow Y_{n}^{\prime}$ satisfying $\psi_{n}^{\prime} f_{n}^{\prime} \sim f_{n-1} \varphi_{n}^{\prime}$.

Consider the following diagram

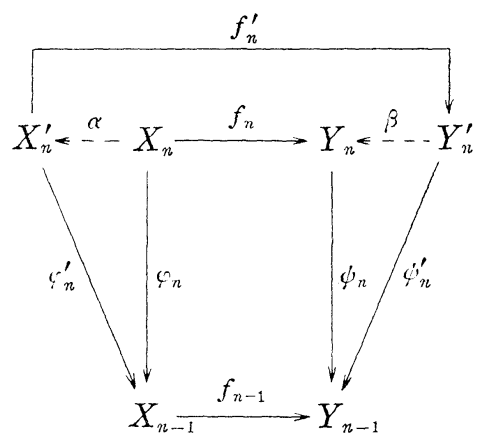

There exist homotopy equivalences $\alpha: X_{n} \rightarrow X_{n}^{\prime}, \beta: Y_{n}^{\prime} \rightarrow Y_{n}$ so $\varphi_{n}^{\prime} \alpha \sim$ $\varphi_{n}$ and $\psi_{n} \beta \sim \psi_{n}^{\prime}$. The map $\beta f_{n}^{\prime} \alpha$ is a lift of $f_{n-1}$, hence the finiteness of the group $\pi_{*}$ (fiber $\psi$ ) and the number of stages implies the finiteness of $M / \sim$. 
Lemma 1.2. Let $f: X \rightarrow Y$ and $f^{\prime}: X^{\prime} \rightarrow Y^{\prime}$ be as in Lemma 1.1, and let $M$ and $M^{\prime}$ be simple connected $C W$-complexes with $H_{*}(M, Z)$ and $H_{*}\left(M^{\prime}, Z\right)$ finite dimensional and finite. Define $S$ to be the set of homotopy classes of pairs $(\varphi, \psi)$ satisfying:

(a) $\varphi: X \rightarrow X^{\prime}$ and $\psi: Y \rightarrow Y^{\prime}$ are maps satisfying $X^{\prime} U$ Cone $U(\varphi)$ is homotopy equivalent to $M$ and $Y^{\prime} \cup$ Cone $(\psi)$ is homotopy equivalent to $M^{\prime}$.

(b) $f^{\prime} \varphi \sim \psi f$.

Then Aut $\left(f^{\prime}\right)$ acts on $S$ and $S /$ Aut $\left(f^{\prime}\right)$ is a finite set.

Proof. Dual to the proof of 1.1 .

THEOREM 1.3. Let $X, Y, X^{\prime}, Y^{\prime}$ be simply connected finite type $C W$-spaces which are either $H_{*}$-finite dimensional or $\pi_{*}$ finite dimensional, and let $f: X \rightarrow Y$ and $f^{\prime}: X^{\prime} \rightarrow Y^{\prime}$ be fibrations.

Suppose $\varphi: X \rightarrow X^{\prime}$ and $\psi: Y \rightarrow Y^{\prime}$ are rational equivalences satisfying $f^{\prime} \varphi \sim$ iff. Then $\operatorname{Aut}(f)$ and $\operatorname{Aut}\left(f^{\prime}\right)$ are commensurable groups.

Proof. Let $\Delta(\varphi, \psi) \subseteq \operatorname{Aut}(f) \times \operatorname{Aut}\left(f^{\prime}\right)$ be the set of pairs $((h, k)$, $\left.\left(h^{\prime}, k^{\prime}\right)\right) \in \operatorname{Aut}(f) \times \operatorname{Aut}\left(f^{\prime}\right)$ for which the diagram

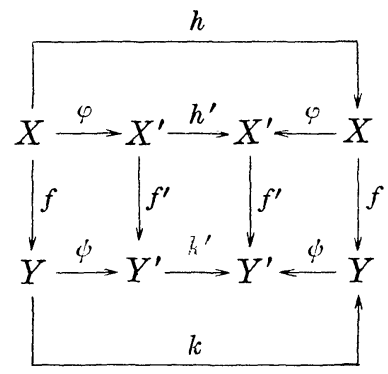

commutes, and let $\operatorname{Stab}\left(\varphi, \psi, \operatorname{Aut}\left(f^{\prime}\right)\right)$ be the image in $\operatorname{Aut}\left(f^{\prime}\right)$ of the second projection map on $\Delta(\varphi, \psi)$. We shall show that $\operatorname{Aut}(f)$ and $\operatorname{Aut}\left(f^{\prime}\right)$ are commensurable with $\Delta(\varphi, \psi)$.

Let $S^{\prime}$ be the set of homotopy classes of pairs of the form $\left(h^{\prime} \varphi h, k^{\prime} \psi k\right)$ where $(h, k) \in \operatorname{Aut}(f)$ and $\left(h^{\prime}, k^{\prime}\right) \in \operatorname{Aut}\left(f^{\prime}\right)$. Then $S^{\prime}$ is a subset of $S$ of Lemma 1.1, and hence $S^{\prime} / \operatorname{Aut}(f)$ is a finite set. But $\operatorname{Aut}\left(f^{\prime}\right)$ acts on $S^{\prime} / \operatorname{Aut}(f)$, i.e., there is a map

$$
\eta: \operatorname{Aut}\left(f^{\prime}\right) \longrightarrow \operatorname{Aut}\left(S^{\prime} / \operatorname{Aut}(f)\right) \text {. }
$$

Then the group $\operatorname{Stab}\left(\varphi, \psi, \operatorname{Aut}\left(f^{\prime}\right)\right)$ contains the kernel of $\eta$, and therefore the fact that $\operatorname{Aut}\left(S^{\prime} / \operatorname{Aut}(f)\right)$ is a finite set implies that $\operatorname{Stab}\left(\varphi, \psi, \operatorname{Aut}\left(f^{\prime}\right)\right)$ has finite index in $\operatorname{Aut}\left(f^{\prime}\right)$. 
On the other hand, the fact that $\varphi$ and $\psi$ are rational equivalences implies that the kernel of the map $\Delta(\varphi, \psi) \rightarrow \operatorname{Aut}\left(f^{\prime}\right)$ is finite. Hence $\Delta(\varphi, \psi)$ and $\operatorname{Aut}\left(f^{\prime}\right)$ are commensurable groups. The proof that $\Delta(\varphi, \psi)$ and $\operatorname{Aut}(f)$ are commensurable is dual.

Notation. For a fibration $f: X \rightarrow Y$ denote by $\operatorname{Aut}_{X}(f)$ the group of homotopy classes of self homotopy equivalences $k: Y \rightarrow Y$ satisfying $k f \sim f$, and by $\operatorname{Aut}_{Y}(f)$ the group of homotopy classes of self homotopy equivalences $h ; X \rightarrow X$ which satisfy $f h \sim f$.

Corollary 1.4. Let $f, f^{\prime}, \varphi$ and $\psi$ be as in Theorem 1.3. Then $\operatorname{Aut}_{X}(f)$ is commensurable with $\operatorname{Aut}_{X^{\prime}}\left(f^{\prime}\right)$ and $\operatorname{Aut}_{Y}(f)$ is commensurable with Aut $_{Y^{\prime}}\left(f^{\prime}\right)$.

TheOREM 1.5. Let $X, Y, X^{\prime}, Y^{\prime}$ be simply connected finite type $C W$-spaces and let $f: X \rightarrow Y$ and $f^{\prime}: X^{\prime} \rightarrow Y^{\prime}$ be fibrations. Suppose $f_{0}$ is homotopy equivalent to $f_{0}^{\prime}$. Then $\operatorname{Aut}(f)$ and $\operatorname{Aut}\left(f^{\prime}\right)$ are commensurable groups.

Proof. Since $f_{0}$ is homotopy equivalent to $f_{0}^{\prime}$ there exists a commutative diagram.

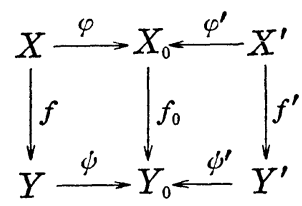

where the horizontal maps are rationalizations.

Let $Y^{\prime \prime}$ be a simply connected $C W$-complex which satisfies: There exist rational equivalences $\alpha: Y^{\prime \prime} \rightarrow Y, \alpha^{\prime}: Y^{\prime \prime} \rightarrow Y^{\prime}$ and $\psi^{\prime \prime}: Y^{\prime \prime} \rightarrow Y_{0}$ so that the following diagram commutes:

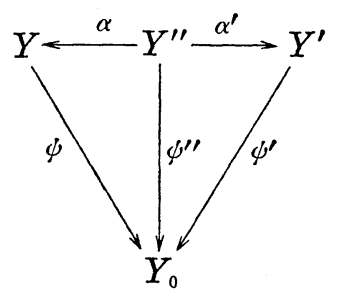

(By Wilkerson [8] such a space exists.)

Consider the following diagram: 


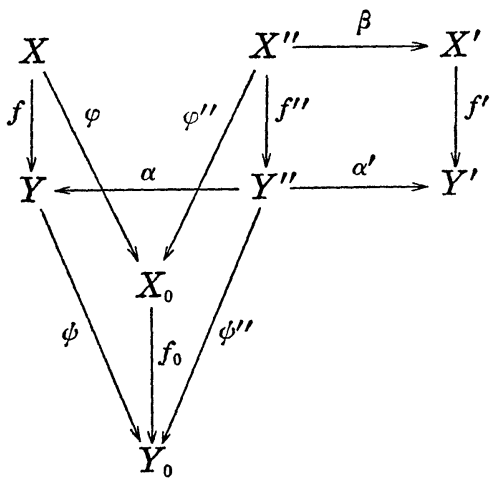

where $X^{\prime \prime}$ is the pullback of $Y^{\prime \prime} \stackrel{\alpha}{\rightarrow} Y^{\prime} \stackrel{f^{\prime}}{\rightarrow} X^{\prime}$ and $\varphi^{\prime \prime}: X^{\prime} \rightarrow X_{0}$ is a rationalization, which satisfies $f_{0} \varphi^{\prime \prime} \sim \psi^{\prime \prime} f^{\prime \prime}$. (The existence of such a rational equivalence follows from the above three diagrams.)

Since $\varphi$ and $\varphi^{\prime \prime}$ aae rationalizations there exists a bouquet of spheres $V S^{n_{i}}$ and maps $X \stackrel{\gamma}{\leftarrow} V S^{n_{i}} \stackrel{\gamma^{\prime \prime}}{\rightarrow} X^{\prime \prime}$ so that $\pi_{*} \gamma \otimes Q$ and $\pi_{*} \gamma^{\prime \prime} \otimes Q$ are epimorphisms and $\phi^{\prime \prime} \gamma^{\prime \prime} \sim \phi \gamma$. Therefore the commutativity of the two parallelograms and the triangle, in the last diagram, implies that $\psi \alpha f^{\prime \prime} \gamma^{\prime \prime} \sim \psi f \gamma$. Consequently there exists a map $\delta: V S^{n_{i}} \rightarrow V S^{n_{i}}$ so that $\alpha f^{\prime \prime} \gamma^{\prime \prime} \delta \sim f \gamma \delta$.

Consider the cofibration $V S^{n_{i}} \stackrel{\lambda}{\rightarrow} V S^{n_{i}} \stackrel{j}{\leftarrow} C_{\lambda}$ where $\operatorname{Im}\left(\pi_{*} \lambda \otimes Q\right)=$ $\operatorname{Ker}\left(\pi_{*}(\gamma \delta) \otimes Q\right)=\operatorname{Ker}\left(\pi_{*}\left(\gamma^{\prime \prime} \delta\right) \otimes Q\right)$. There exist maps $\varepsilon: C_{\chi} \rightarrow X$, $\varepsilon^{\prime \prime}: C_{\lambda} \rightarrow X^{\prime \prime}$ so that $\varepsilon j \sim \gamma \delta, \varepsilon^{\prime \prime} j \sim \gamma^{\prime \prime} \delta$ and $\varphi^{\prime \prime} \varepsilon^{\prime \prime} \sim \varphi \varepsilon$. Consequently the considerations of the previous paragraph imply the existence of a map $\mu: V S^{n_{i}} \rightarrow V S^{n_{i}}$ and rational equivalences $\phi: C_{\lambda \mu} \rightarrow X, \phi^{\prime \prime}: C_{\lambda \mu} \rightarrow X^{\prime \prime}$ $\left(C_{\lambda \mu}\right.$ - the cofibre of $\left.\lambda \mu\right)$, so that $\alpha f^{\prime \prime} \phi^{\prime \prime} \sim f \phi$. Hence Theorem 1.3 implies that $\operatorname{Aut}(f)$ and $\operatorname{Aut}\left(f^{\prime}\right)$ are both commensurable with $\operatorname{Aut}\left(f^{\prime \prime} \phi^{\prime \prime}\right)$ and therefore they are commensurable.

2. Proof of the main theorem. By Wilkerson [8] there are finitely generated free simplicial $N^{c} Z$ groups $M$. an $N$. and a map $f .: M . \rightarrow N$. so that Aut $(f)$ can be identified with the group of loop homotopy equivalence classes of self-equivalences of $f$, and $\operatorname{Aut}\left(f_{0}\right)$ can be identified with the group of loop homotopy equivalence classes of self-equivalences of $f_{0}:: M_{0}$. $\rightarrow N_{0}$. Theretore we study here these groups. We denote them by $H \operatorname{Aut}(f)$ and $H \operatorname{Aut}\left(f_{0}\right)$, respectively.

Let $M_{0}$. and $N_{0}$. be finitely generated $N^{c} Q$ groups. Denote by $\left.\operatorname{Aut}\left(M_{0} \cdot\right)_{0} \operatorname{Aut}\left(N_{0} \cdot\right)_{0}\right)$ the group of simplicial automorphisms of $M_{0} \cdot\left(N_{0} \cdot\right)$ and by $\operatorname{Aut}\left(M_{0}\right)_{1}\left(\operatorname{Aut}\left(N_{0} \cdot\right)_{1}\right.$ the set of automorphisms of $M_{0} \otimes \Delta(1)$ $\left(N_{0} \cdot \otimes \Delta(1)\right)$ lying over the identity on $\Delta(1)$. The face maps $d_{0}, d_{1}$ : $\operatorname{Aut}\left(M_{0} \cdot\right)_{1} \rightarrow \operatorname{Aut}\left(M_{0} \cdot\right)_{0}$ and $d_{0}^{\prime}, d_{1}^{\prime}: \operatorname{Aut}\left(N_{0} \cdot\right)_{1} \rightarrow \operatorname{Aut}\left(N_{0} \cdot\right)_{0}$.

Let $\operatorname{SimpAut}\left(f_{0}\right.$. $)$ denote the set of simplicial automorphisms of 
$f_{0}$. Two pair $(h, k),\left(h^{\prime}, k^{\prime}\right) \in \operatorname{SimpAut}\left(f_{0}\right)$ are homotopic if and only if $h^{\prime} \in d_{1} d_{0}^{-1}(h)$ and $k^{\prime} \in d_{1}^{\prime} d_{0}^{\prime-1}(k)$. Hence

$$
H \operatorname{Aut}\left(f_{0}\right)=\operatorname{Simp} \operatorname{Aut}\left(f_{0}\right) /\left(d_{1} d_{0}^{-1}(i d) \times d_{1}^{\prime} d_{0}^{\prime-1}(i d)\right) \cap \operatorname{Simp} \operatorname{Aut}\left(f_{0} .\right) .
$$

Proposition 2.1. Let $f_{0}: M_{0} \rightarrow N_{0}$. be a simplicial map between finitely generated free simplicial $N^{c} Q$ groups. There exists an affine group scheme $G$ over $Q$, so that $\operatorname{Simp} \operatorname{Aut}\left(f_{0}.\right)$ can be identified with the $Q$-valued points of $G$.

Proof. Similar to the proof of Proposition 9.2 in Wilkerson [8].

Proposition 2.2. There is a normal closed subgroup scheme over $Q, H$ of $G$, such that $\left(d_{1} d_{0}^{-1}(i d) \times d_{1}^{\prime} d_{0}^{\prime-1}(i d)\right) \cap \operatorname{SimpAut}\left(f_{0}\right)=H(Q)$.

Proof. Since linear algebraic groups are closed under finite cartezian products and finite intersections, the result follows from Proposition 9.3 in Wilkerson [8].

Proposition 2.3. Let $G$ and $H$ be as defined above. There exists an affine group scheme $G / H$ over $Q$, such that $H \operatorname{Aut}\left(f_{0}\right)=(G / H)(Q)=$ $G(Q) / H(Q)$.

Proof. Proposition 9.4. in Wilkerson [8], the discussion above and thefact that a subgroup of a unipotent group is unipotent, implies that $H$ is unipotent and that $H \operatorname{Aut}\left(f_{0}\right)=G(Q) / H(Q)$. By Borel $[1,6.8]$, the quotient of an affine group scheme over $Q$ by a closed normal subgroup scheme over $Q$ is again an affine group scheme over $Q$. That is $G / H$ exists. The Galois cohomology sequence [Serre] $1 \rightarrow$ $H(Q) \rightarrow G(Q) \rightarrow G / H(Q) \rightarrow H^{1}(\operatorname{Gal}(\bar{Q}, Q), H) \cdots$ is an exact sequence of groups and pointed sets. Hence the fact that $H$ is unipotent implies that $H^{1}(\operatorname{Gal}(\bar{Q}, Q), H)=0$ and the result follows.

Proposition 2.3'. Let $X, Y$ be simply connected finite $C W$ complexes and let $f: X \rightarrow Y$ be a fibration. Then $\operatorname{Aut}\left(f_{0}\right)$ is the set of Q-valued points of a linear algebraic group over $Q$.

Proposition 2.4. Let $M$. and $N$. be finitely generated free simplicial nilpotent groups of class $c$ and let $f: M . \rightarrow N$. be a simplicial map. Define $M_{L} \subseteq M_{0}\left(N_{L} \subseteq N_{0}\right)$ to be the intersection of all lattice subgroups of $M_{0} .\left(N_{0}.\right)$ that contain $M .(N$.$) .$

Then $f$. induces a map $f_{L}: M_{L} \rightarrow N_{L}$ and Simp Aut(f.) has finite index in $\operatorname{Simp} \operatorname{Aut}\left(f_{L}\right)$. 
Proof. The existence of $f_{L}$ and the fact that $G_{\overline{\text { def }}}(\operatorname{Simp} \operatorname{Aut}(M) \times$. $\operatorname{SimpAut}(N).) \cong \operatorname{SimpAut}\left(M_{L}\right) \times \operatorname{Simp} \operatorname{Aut}\left(N_{L}\right)$ is a subgroup of finite index, follows from Wilkerson [8, 8.1 and 8.3]. Hence

$$
G \cap \operatorname{SimpAut}\left(f_{L}\right) \cong \operatorname{SimpAut}\left(f_{L}\right)
$$

is a subgroup of finite index and it suffices to prove that $\operatorname{SimpAut}\left(f_{L}\right)=$ $G \cap \operatorname{SimAut}\left(f_{L}\right)$. But this is clear, since $(h, k) \in G \cap \operatorname{SimpAut}\left(f_{L}\right)$ implies that $h|M .: M . \rightarrow M ., k| N .: N . \rightarrow N$. and $k f_{L} h^{-1}=f_{L}$ and therefore $\left(h\left|M_{.}, k\right| N_{\text {. }}\right) \in \operatorname{Simp} \operatorname{Aut}(f$.).

Proposition 2.5. Let $X, Y$ be simply connected finite $C W$ complexes and let $f: X \rightarrow Y$ be a fibration. There exist finite $C W$ complexes $X^{\prime}$ and $Y^{\prime}$ so that $H_{*}\left(X^{\prime}, Z\right)$ and $H_{*}\left(Y^{\prime}, Z\right)$ are torsion free and a fibration $f^{\prime}: X^{\prime} \rightarrow Y^{\prime}$ so that $\operatorname{Aut}\left(f^{\prime}\right)$ and $\operatorname{Aut}(f)$ are commensurable groups.

Proof. By Theorem 1.3 it suffices to prove that there exist rational equivalences $h: X^{\prime} \rightarrow X$ and $k: Y^{\prime} \rightarrow Y$ so that the diagram

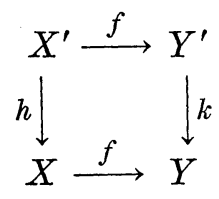

commutes.

Since $f$ is homotopic to a cellular map we can assume that $f$ is cellular. Suppose there exists a commutative diagram

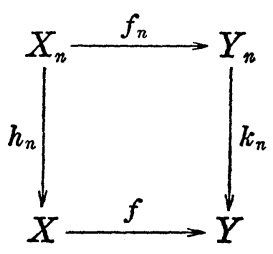

where $f_{n}$ is cellular, $h_{n}, k_{n}$ are rational equivalences and the groups $H_{m}\left(X_{n}, Z\right)$ and $H_{m}\left(Y_{n}, Z\right)$ are torsion free for $m \leqq n$.

Let $X_{n}^{(n)}, Y_{n}^{(n)}$ be the $n$-skeletons of $X_{n}$ and $Y_{n}$. Since $f_{n}$ is cellular $f_{n}$ induces a map $f_{n}^{\prime}: X_{n} / X_{n}^{(n)} \rightarrow Y_{n} / Y_{n}^{(n)}$. Therefore the fact that $H_{n+1}(X, Z)=\pi_{n+1}\left(X_{n} / X_{n}^{(n)}\right)$ and $H_{n+1}(Y, Z)=\pi_{n+1}\left(Y_{n} / Y_{n}^{(n)}\right)$ implies the existence of a commutative diagram ${ }^{t}\left({ }^{t}\right)$ denotes the torsion subgroup of ( ).) 


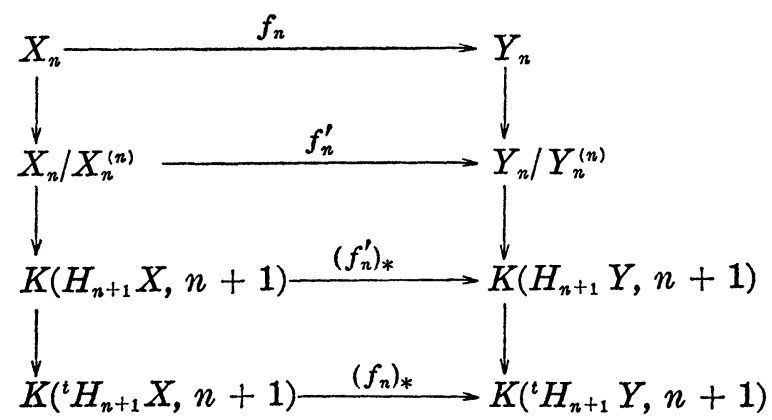

Let $X_{n+1}$ and $Y_{n+1}$ be the fibers of the maps

$$
X_{n} \longrightarrow X_{n} / X_{n}^{(n)} \longrightarrow K\left(H_{n+1} X, Z\right) \longrightarrow K\left({ }^{t} H_{n+1} X, Z\right)
$$

and

$$
Y_{n} \longrightarrow Y_{n}^{(n)} / Y_{n}^{(n)} \longrightarrow K\left(H_{n+1} Y, Z\right) \longrightarrow K\left(t_{n+1} Y, Z\right)
$$

and let $f_{n+1}: X_{n+1} \rightarrow Y_{n+1}$ be the iduced map. Obviously $X_{n+1}$ is rational equivalent to $X, Y_{n+1}$ is rational equivalent to $Y$ and there exists a commutative diagram

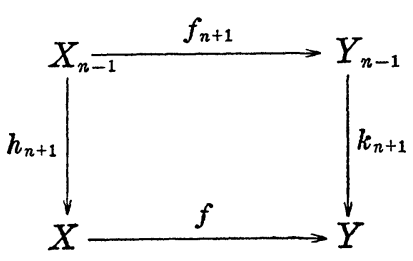

where $h_{n+1}$ and $k_{n+1}$ are rational equivalences and $f_{n+1}$ is cellular. By the Serre spectral sequence $H_{m}\left(X_{n+1}, Z\right)$ and $H_{m}\left(Y_{n+1}, Z\right)$ are torsion free for $m \leqq n+1$, and the result follows.

Proposition 2.6. Let $M$. and $N$. be finitely generatad connected minimal simplicial $N^{c} Z$ groups and let $f: M . \rightarrow N$. be a simplicial map. Then $H \operatorname{Aut}(f$.$) is an arithmetic subgroup of H \operatorname{Aut}\left(f_{0}\right)$.

Proof. Since $\operatorname{Simp} \operatorname{Aut}(f) \subseteq \operatorname{Simp} \operatorname{Aut}\left(f_{L}\right)$ is a subgroup of finite index, the theorem follows from Theorem 9.8 in [8] by replacing $N$. by $f ., N_{L}$ by $f_{L}$ and $d_{1} d_{0}^{-1}(i d)$ by $\left(d_{1} d_{0}^{-1}(i d) \times d_{1}^{\prime} d_{0}^{\prime-1}(i d) \cap \operatorname{Simp} \operatorname{Aut}\left(f_{0}\right)\right.$.

Proof of the main theorem. By Proposition 2.5 we can assume that $H_{*}(X, Z)$ and $H_{*}(Y, Z)$ are torsion free. Hence by Wilkerson [8] $\operatorname{Aut}(f)$ can be calculated as $H \operatorname{Aut}(f$.) for some $f:: M$. $\rightarrow N$., where $M$. and $N$. are connected minimal free simplicial $N^{\circ} Z$ groups. Therefore $\operatorname{Aut}(f)$ is an arithmetic subgroup of a linear algebraic group, and the result follows from Proposition 10.3 in [8]. 
CoROLlaRy 2.7. Let $X, Y$ be simply connected finite $C W$ complexes and let $f: X \rightarrow Y$ be a fibration. Then $\operatorname{Aut}_{Y}(f)$ is finitely presented.

Proof. Similar to the proof of the main theorem.

CoROllary 2.8. Let $X$ be a simply connected finite $C W$-complex and let $G \subseteq \operatorname{Aux} X$ be a finitely generated subgroup. If $H_{*}(X, Z)$ is torsion free then the centralizer of $G$ is finitely presented.

Proof. Suppose $G$ is generated by $g_{1}, g_{2}, \cdots, g_{n}$. Since the centralizer of $G$ is equal to the centralizer of the set $\left\{g_{1}, g_{2}, \cdots, g_{n}\right\}$, the proof is similar to the proof of the main theorem.

3. Commensurability and $H_{0}$-spaces and fibrations. Let $X, Y$ be $H_{0}$-spaces and let $f: X \rightarrow Y$ be a fibration. In this section we deal with the relation between Aut $X$ and Aux $H^{*}(X, Y)$ and between Aut $(f)$ and Aut $H^{*}(f, Z)$. In case $X$ is an $H$-space we draw conclusions on the relation between the $H$-structures on $X$ and the Hopfalgebra structures on $H^{*}(X, Q)$.

Notation. For any $H_{0}$-space $X$ we denote $K\left(Q H^{*}(X, Z) /\right.$ torsion $)$ by $K(X)$.

Proposition 3.1. Let $f_{1}, f_{2}: X \rightarrow Y$ be fibrations. If $\operatorname{rank}\left(H^{*}\left(f_{1}, Q\right)\right)$ is equal to $\operatorname{rank}\left(H^{*}\left(f_{2}, Q\right)\right)$ then $\operatorname{Aut}\left(f_{1}\right)$ and $\operatorname{Aut}\left(f_{2}\right)$ are commensurable groups.

Proof. Since $\operatorname{rank}\left(H^{*}\left(f_{1}, Q\right)\right)=\operatorname{rank}\left(H^{*}\left(f_{2}, Q\right)\right)$ there exist Eilenberg-Maclane spaces $K_{1}, K_{2}$ and rational equivalences $\varphi_{i}: X \rightarrow$ $K(X), \psi_{i}: Y \rightarrow K(Y)(i=1,2)$ so that $K(X)=K \times K_{1}, K(Y)=K \times K_{2}$ and the following diagram commutes

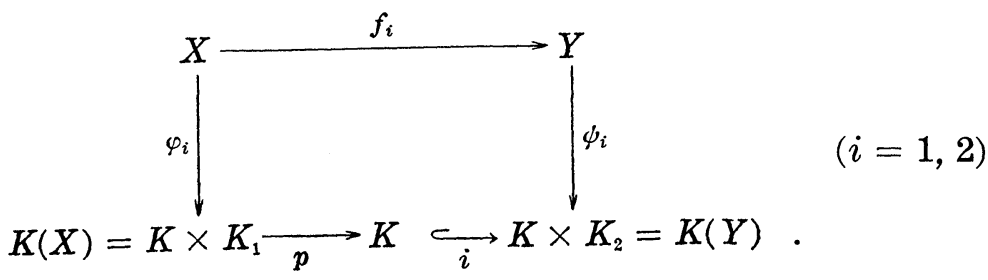

Hence $\operatorname{Aut}(f)$ and $\operatorname{Aut}(g)$ are both commensurable with $\operatorname{Aut}(i p)$ and therefore they are commensurable groups.

Proposition 3.2. Let $X, Y$ be $H_{0}$-spaces and let $f: X \rightarrow Y$ be a fibration. Then: 
(a) The $\operatorname{map}[Y, X] \rightarrow \operatorname{Hom}\left(H_{*}(Y, Z), H_{*}(X, Z)\right)$ is finite to one.

(b) $\operatorname{Im}\left(\right.$ Aut $X \rightarrow$ Aut $H^{*}(X, Z)$ ) is a subgroup of finite index.

(c) The kernel of the obvious map $\eta$ : Aut $(f) \rightarrow$ Aut $H^{*}(f, Z)$ is finite and its image is a subgroup of finite index in Aut $H^{*}(f, Z)$.

(d) For any pair $(h, k) \in$ Aut $H^{*}(f, Z)$ there exists a pair $(\widetilde{h}, \widetilde{k}) \in$ Aut $(f)$ and an integer $m$, so that $H^{*}(\widetilde{h}, Z)=h^{m}$ and $H^{*}(\widetilde{k}, Z)=k^{m}$.

Proof. (a) Let $\varphi: X \rightarrow K(X)$ be a rational equivalence which represents generators of $H^{*}(X, Z) /$ torsion. Since $H^{*}(f, Z)=H^{*}(g, Z)$ $(f, g: Y \rightarrow X)$ implies that $\varphi f \sim \varphi g$, the result follows from the fach that any map $h: Y \rightarrow K(X)$ has only a finite number of lifts to a map $\tilde{h}: Y \rightarrow X$, which satisfies $\varphi \widetilde{h} \sim h$.

(b) Let $\varphi: X \rightarrow K(X)$ be as in (a). By Wilkerson [8] $\operatorname{Im}(\operatorname{Aut}(\varphi) \stackrel{p r o j}{\longrightarrow}$ $\operatorname{Aut}(K(X)) \rightarrow \operatorname{Aut}\left(H^{*}(K(X), Z) /\right.$ torsion $) \rightarrow \operatorname{Aut}\left(H^{*}(X, Z) /\right.$ torsion $)$ is a subgroup of finite index in $\operatorname{Aut}\left(H^{*}(X, Z) /\right.$ torsion $)$. Hence the result follows from the fact that $\operatorname{Im}\left(\operatorname{Aut} X \rightarrow \operatorname{Aut}\left(H^{*}(X, Z) /\right.\right.$ torsion $)$ ) contains the image of the above map.

(c) The fact that Kern is a finite group follows from part (a).

Let $G=\operatorname{Im}\left(\right.$ Aut $X \times$ Aut $Y \rightarrow$ Aut $H^{*}(X, Z) \times$ Aut $H^{*}(Y, Z)$ ). By part (b) $G$ is a subgroup of finite index in Aut $H^{*}(X, Z) \times \operatorname{Aut} H^{*}(Y, Z)$, hence $G \cap$ Aut $H^{*}(f, Z) \subseteq$ Aut $H^{*}(f, Z)$ is a subgroup of finite index and it suffices to prove that $\operatorname{Im} \eta \subseteq G \cap \operatorname{Aut} H^{*}(f, Z)$ is a subgroup of finite index.

Let $(h, k) \in G \cap$ Aut $H^{*}(f, Z)$. There exists a pair $(\tilde{h}, \widetilde{k}) \in$ Aut $X \times$ Aut $Y$ satisfying $H^{*}(\widetilde{h}, Z)=h, H^{*}(\widetilde{k}, Z)=k$ and $H^{*}\left(\widetilde{k^{-1}} f \widetilde{h}, Z\right)=$ $H^{*}(f, Z)\left(\tilde{k}^{-1}-\right.$ the homotopy inverse of $\left.\widetilde{k}\right)$. Therefore the fact that there is only a finite number of maps $f_{1}, f_{2}, \cdots, f_{n}$ which satisfy $H^{*}\left(f_{i}, Z\right)=H^{*}(f, Z)$ implies that $\operatorname{Im} \eta \subseteq G \cap \operatorname{Aut} H^{*}(f, Z)$ is a subgroup of finite index, and the proof of part (c) is complete.

(d) Suppose $(h, k) \in$ Aut $H^{*}(f, Z)$. We have to show that there exists an integer $m$ so that $\left(h^{m}, k^{m}\right) \in \operatorname{Im}\left(\operatorname{Aut}(f) \rightarrow\right.$ Aut $\left.H^{*}(f, Z)\right)$. Since $h$ and $k$ are automorphisms, this follows immediately from the fact that $\operatorname{Im}\left(\operatorname{Aut}(f) \rightarrow \operatorname{Aut} H^{*}(f, Z)\right)$ is a subgroup of finite index in Aut $H^{*}(f, Z)$.

Corollary 3.3. Let $X$ be an $H_{0}$-space. Suppose $h, k \in$ Aut $X$ satisfy $H^{*}(h, Z)=H^{*}(k, Z)$. Then there exists an integer $m$ so that $h^{m} \sim k^{m}$. Consequently $h \in$ Aut $X$ is of finite order if and only if $H^{*}(h, Z)$ is.

Proof. The pair $\left(H^{*}(h, Z), H^{*}(k, Z)\right) \in \operatorname{Aut}\left(H^{*}\left(1_{X}, Z\right)\right)$, hence the result follows from part (d) in Proposition 3.2.

Corollary 3.4. Suppose $X, Y$ are $H_{0}$-spaces, $f: X \rightarrow Y$ a fibration 
and $(h, k) \in \operatorname{Aut}(f)$. Then:

(a) $H^{*}(f, Z)$ is monic and the order of $h$ is finite implies that the order of $k$ is finite.

(b) $H^{*}(f, Z)$ is epic and the order of $k$ is finite implies that the order of $h$ is finite.

Proof. (a) Obviously, the order of $h$ is finite implies that the order of $H^{*}(k, Z)$ is finite. Hence the result follows from Corollary 3.3 .

(b) Similar to (a).

Corollary 3.5. Let $X, Y$ and $f$ be as in Corollary 3.4. Then: (a) $H^{*}(f, Z)$ is monic and Aut $X$ is finite implies that Aut $(f)$ is finite.

(b) $H^{*}(f, Z)$ is epic and Aut $Y$ is finite implies that $\operatorname{Aut}(f)$ is finite.

Proof. (a) $\left(h, k_{1}\right),\left(h, k_{2}\right) \in \operatorname{Aut}(f)$ and $H^{*}(f, Z)$ is monic implies that $H^{*}\left(k_{1}, Z\right)=H^{*}\left(k_{2}, Z\right)$. Therefore the fact that the kernel of the map Aut $Y \rightarrow$ Aut $H^{*}(f, Z)$ is finite implies that for each $h \in$ Aut $X$ there exist, at most, a finite number of $k \in A u t Y$, so that the pair $(h, k) \in \operatorname{Aut}(f)$. Hence $\operatorname{Aut}(f)$ is a finite group.

(b) Similar to (a).

In order to draw conclusions from Proposition 3.2 to the case that $X$ is an $H$-space we need the following definitions:

Definition. Let $X$ be an $H$-space and let $\mu_{1}, \mu_{2}$ be two $H$ structures on $X$.

(a) We say that $\mu_{1}$ is equivalent to $\mu_{2}$ if there exists a homotopy equivalence $h: X \rightarrow X$, so that $h \mu_{1} \sim \mu_{2}(h \times h)$.

(b) We say that $H^{*}\left(\mu_{1}, Z\right) /$ torsion is equivalent to $H^{*}\left(\mu_{2}, Z\right) /$ torsion if there exists a map $h \in \operatorname{Aut}\left(H^{*}(X, Z) /\right.$ torsion $)$ so that

$$
\left(h_{*} \otimes h_{*}\right) H^{*}\left(\mu_{1}, Q\right)=H^{*}\left(\mu_{2}, Q\right) h_{*} \text {. }
$$

(c) We say that $H^{*}\left(\mu_{1}, Q\right)$ is equivalent to $H^{*}\left(\mu_{2}, Q\right)$ if there exists a map $h \in H^{*}(X, Q)$ so that $(h \otimes h) H^{*}\left(\mu_{1}, Q\right)=H^{*}\left(\mu_{2}, Q\right) h$.

Proposition 3.6. Let $X, \mu_{0}$ be an $H$-space. Then the number of equivalence classes of $H$-structures $\mu$ on $X$, for which $H^{*}(\mu, Z) /$ torsion is equivalent to $H^{*}\left(\mu_{0}, Z\right) /$ torsion is finite.

Proof. Let $\eta$ : Aut $X \rightarrow$ Aut $H^{*}(X, Z) /$ torsion be the obvious map. By Proposition $3.2(\mathrm{~b}) \operatorname{Im} \eta \subseteq$ Aut $H^{*}(X, Z) /$ torsion is a subgroup of 
finite index. Assume that the index is $n$ and that $h_{1}, h_{2}, \cdots, h_{n-1} \in$ $\operatorname{Aut}\left(H^{*}(H, Z) /\right.$ torsion $)$ satisfy

$\operatorname{Aut}\left(H^{*}(X, Z) /\right.$ torsion $)=\operatorname{Im} \eta \cup h_{1} \operatorname{Im} \eta \cup \cdots \cup h_{n-1} \operatorname{Im} \eta$.

Let $\mu_{1}, \mu_{2}$ be $H$-structures on $X$ and let $h, h^{\prime} \in \operatorname{Aut}\left(H^{*}(X, Z)\right) /$ torsion satisfy:

$$
H^{*}\left(\mu_{0}, Q\right)\left(h_{i} h\right)_{*}=\left(\left(h_{i} h\right)_{*} \otimes\left(h_{i} h\right)_{*}\right) H^{*}\left(\mu_{1}, Q\right),
$$

and

$$
H^{*}\left(\mu_{0}, Q\right)\left(h_{i} h^{\prime}\right)_{*}=\left(\left(h_{i} h^{\prime}\right)_{*} \otimes\left(h_{i} h^{\prime}\right)_{*}\right) H^{*}\left(\mu_{2}, Q\right),
$$

where $h=H^{*}(\widetilde{h}, Z) /$ torsion and $h^{\prime}=H^{*}\left(\tilde{h}^{\prime}, Z\right) /$ torsion. Then:

$$
H^{*}\left(\mu_{2}, Z\right) / \text { torsion }=H^{*}\left(\widetilde{h}^{\prime} \widetilde{h}^{-1} \mu_{1}\left(\widetilde{h}^{\prime} \widetilde{h}^{-1} \times \widetilde{h}^{\prime} \widetilde{h}^{-1}\right)\right) / \text { torsion i.e., } \mu_{1}
$$

is equivalent to an $H$-structure $\mu^{\prime}$ which satisfies $H^{*}\left(\mu^{\prime}, Z\right) /$ torsion $=$ $H^{*}\left(\mu_{2}, Z\right) /$ torsion. Consequently the results follows from the fact that for any $H$-structure $\mu$ on $X$, the number of $H$-structures $\mu^{\prime}$ which satisfy $H^{*}\left(\mu^{\prime}, Z\right) /$ torsion $=H^{*}(\mu, Z) /$ torsion is finite (this follows from Proposition 3.2(a)).

Proposition 3.7. Let $X, \mu_{0}$ be an $H$-space. Suppose $H^{*}\left(\mu_{0}, Q\right)$ is primitively generated, then the number of equivalence classes of $H$-structures $\mu$ on $X$ for which $H^{*}(\mu, Q)$ is equivalent to $H^{*}\left(\mu_{0}, Q\right)$ is finite.

Proof. By Proposition 3.6 the number of equivalence classes of $H$-structures $\mu$ on $X$, for which $H^{*}(\mu, Z) /$ torsion is equivalent to $H^{*}\left(\mu_{0}, Z\right) /$ torsion is finite. Hence it suffices to prove that the number of equivalence classes of comultiplications $H^{*}(\mu, Z) /$ torsion $(\mu: X \times X \rightarrow$ $X$ an $H$-structure) for which $H^{*}(\mu, Q)$ is equivalent to $H^{*}\left(\mu_{0}, Q\right)$ is finite.

Let $A$ be the set of the comultiplications $\nu: H^{*}(X, Z) /$ torsion $\rightarrow$ $H^{*}(X, Z) /$ torsion $\otimes H^{*}(X, Z) /$ torsion which satisfy: There exists a multiplication $\mu: X \times X \rightarrow X$ so that $\nu=H^{*}(\mu, Z) /$ torsion and $H^{*}(\nu, Q)$ is equivalent to $H^{*}\left(\mu_{0}, Q\right)$. Denote by $\varphi: A \rightarrow \operatorname{Hom}\left(H^{*}(X, Q), H^{*}(X, Q) \otimes\right.$ $\left.H^{*}(X, Q)\right)$ and by $\eta: \operatorname{Aut}\left(H^{*}(X, Z) /\right.$ torsion $) \rightarrow$ Aut $H^{*}(X, Q)$ the obvious maps. Since the kernels of $\varphi$ and $\eta$ are finite it suffices to prove [2, Proof of Theorem I] that the number of equivalence classes of $\operatorname{Im} \varphi$ relative to the equivalence relation: $\varphi\left(\mu_{1}\right) \sim \varphi\left(\mu_{2}\right)$ if and only if there exists $h \in \operatorname{Im} \eta$ so that $\varphi\left(\mu_{1}\right) h=(h \otimes h) \varphi\left(\mu_{2}\right)\left(\mu_{1}, \mu_{2} \in A\right)$ is finite.

By Curjel $[2,5.2]$ the fact that the groups $\operatorname{Hom}\left(H^{*}(X, Z) /\right.$ torsion, $H^{*}(X, Z) /$ torsion $)$ and $\operatorname{Hom}\left(H^{*}(X, Z) /\right.$ torsion, $H^{*}(X, Z) /$ torsion $\otimes$ $H^{*}(X, Z) /$ torsion) are finitely generated implies the existence of a basis $X=\left\{x_{i j}\right\}$, of $P H^{*}\left(X, \mu_{0}, Q\right)$, so that the matrix of every map 
$f \in \operatorname{Hom}\left(H^{*}(X, Z) /\right.$ torsion, $H^{*}(X, Z) /$ torsion $)$, with respect to this basis, is integral, and the matrix of every map

$g \in \operatorname{Hom}\left(H^{*}(X, Z) /\right.$ torsion, $H^{*}(X, Z) /$ torsion $\otimes H^{*}(X, Z) /$ torsion

with respect to the basis $\left\{x_{i j} \otimes 1,1 \otimes x_{i j}\right\}$ of $P H^{*}\left(X \times X, \mu_{0}, Q\right)$ is, also, integral. In particular the matrix of every map which belongs either to $\operatorname{Im} \psi$ or to $\operatorname{Im} \eta$, with respect to the above bases, is integral. Hence the result follows from the following theorem of SamelsonLeray:

Theorem of Samelson-Leray [3, 3 Exp 2]. Let $A$ be an algebra over the integers. Suppose that $A$ has no generators in even dimensions. Then all the associative comultiplications on $A$ are equivalent.

4. Genus and automorphism. Let $X$ and $Y$ be nilpotent $C W$ complexes of finite type and let $f: X \rightarrow Y$ be a fibration. Denote by $G(X)$ the genus of $X$ and by $G(f)$ the genus of $f$.

In this section we investigate the relations between Aut $X$ and Aut $X^{\prime}$ where $X^{\prime} \in G(X)$ and between $\operatorname{Aut}(f)$ and Aut $\left(f^{\prime}\right)$ where $f^{\prime} \in G(f)$.

Notation. Let $X$ be a nilpotent $C W$-complex and let $\varphi: X \rightarrow X_{0}$ be a rationalization. For every prime $p$ and for every $h \in$ Aut $X$ denote by $\left(h_{p}\right)_{\varphi}$ the localization of $h$ at $p$ with respect to $\varphi$.

Proposition 4.1. Let $X$ be a nilpotent $C W$-complex with a finite number of homology groups and let $p \in P(P-$ the set of primes $)$. If $X^{\prime} \in G(X)$ then $\operatorname{Ker}\left(\right.$ Aut $X \rightarrow$ Aut $\left.X_{p}\right)$ is isomorphic to $\operatorname{Ker}\left(\operatorname{Aut} X^{\prime} \rightarrow\right.$ Aut $\left.X_{p}\right)$.

Proof. Let $\varphi: X \rightarrow X_{0}$ and $\psi: X^{\prime} \rightarrow X_{0}$ be rationalizations and let $h \in \operatorname{Ker}\left(\right.$ Aut $X \rightarrow$ Aut $X_{p}$ ). Since for every prime $p$ and for every localization $\phi_{p}: X_{p} \rightarrow X_{0} \phi_{p}\left(h_{p}\right)_{\varphi} \sim 1_{X_{0}} \phi_{p}$, there exists a unique map $h^{\prime} \epsilon$ Aut $X^{\prime}$ so that $\left(h_{p}^{\prime}\right)_{\psi}=\left(h_{p}\right)_{\psi}$ for every prime $p$ [5, II 5.6]. Obviously $h^{\prime} \in \operatorname{Ker}\left(\right.$ Aut $X^{\prime} \rightarrow$ Aut $\left.X_{p}^{\prime}\right)$. Hence the map $\eta: \operatorname{Ker}\left(\right.$ Aut $X \rightarrow$ Aut $\left.X_{p}\right) \rightarrow$ $\operatorname{Ker}\left(\right.$ Aut $\left.X^{\prime} \rightarrow \operatorname{Aux} X_{p}\right)$ defined by $\eta(h)=h^{\prime}$ iff $\left(h_{p}^{\prime}\right)_{\psi}=\left(h_{p}\right)_{\varphi}$ for every prime $p$, is a well defined homomorphism. The same considerations imply the existence of a homomorphism $\eta^{\prime}: \operatorname{Ker}\left(\right.$ Aut $X^{\prime} \rightarrow$ Aut $\left.X_{p}\right) \rightarrow$ $\operatorname{Ker}\left(\right.$ Aut $X \rightarrow$ Aut $\left.X_{p}\right)$ defined by: $\eta^{\prime}(k)=k^{\prime}$ iff $\left(k_{p}^{\prime}\right)_{\varphi}=\left(k_{p}\right)_{\psi}$ for every prime $p$. Since $\eta^{\prime} \eta$ and $\eta \eta^{\prime}$ are identities $\operatorname{Ker}\left(\right.$ Aut $X \rightarrow \operatorname{Aut} X_{p}$ ) is isomorphic to $\operatorname{Ker}\left(\operatorname{Aut} X^{\prime} \rightarrow \operatorname{Aut} X_{p}^{\prime}\right)$.

COROLlary 4.2. Let $X$ be an $H_{0}$-space with either a finite number of homology groups or a finite number of homotopy groups. Then 
for every $X^{\prime} \in G(X) \operatorname{Ker}\left(\right.$ Aut $X \rightarrow$ Aut $H^{*}(X, Q)$ ) is isomorphic to $\operatorname{Ker}\left(\right.$ Aut $X^{\prime} \rightarrow$ Aut $H^{*}\left(X^{\prime}, Q\right)$ ). 4.1 .

Proof. Since $X_{0}=\Pi K\left(Q, n_{j}\right)$ the result follows from Proposition

CoRollary 4.3. If $X$ and $X^{\prime}$ are as in Corollary 4.2 then $\operatorname{Ker}\left(\right.$ Aut $X \rightarrow$ Aut $\left.H_{*}(X, Z)\right)$ is isomorphic to

$$
\operatorname{Ker}\left(\text { Aut } X^{\prime} \longrightarrow \text { Aut } H_{*}\left(X^{\prime}, Z\right)\right) \text {. }
$$

Proof. Let $\eta$ and $\eta^{\prime}$ be as in the proof of Proposition 4.1. We have to show that $\eta\left(\operatorname{Ker}\left(\right.\right.$ Aut $X \rightarrow$ Aut $\left.H_{*}(X, Z)\right) \subseteq \operatorname{Ker}\left(\right.$ Aut $X^{\prime} \rightarrow$ Aut $\left.H_{*}\left(X^{\prime}, Z\right)\right)$ and that $\eta^{\prime}\left(\operatorname{Ker}\left(\right.\right.$ Aut $\left.X^{\prime} \rightarrow \operatorname{Aut} H_{*}\left(X^{\prime}, Z\right)\right) \subseteq \operatorname{Ker}(\operatorname{Aut} X \rightarrow$ Aut $\left.H_{*} X, Z\right)$ ).

Suppose $h \in \operatorname{Ker}\left(\right.$ Aut $X \rightarrow$ Aut $\left.H_{*}(X, Z)\right)$ and $\eta(h)=h^{\prime}$. The definition of $\eta$ and the fact that for every prime $p H_{*}(h, Z) \otimes Z_{(p)}=$ $1\left(Z_{(p)}\right.$ - the localization of $Z$ at $\left.p\right)$ imply for every prime $p H_{*}\left(h^{\prime}, Z\right) \otimes$ $Z_{(p)}=1$. Hence it follows from Hilton-Mislin and Roitberg [5, I. 3.13] that $h^{\prime} \in \operatorname{Ker}\left(\right.$ Aut $X^{\prime} \rightarrow$ Aut $\left.H^{*}\left(X^{\prime}, Z\right)\right)$. The proof that $\eta^{\prime}\left(\operatorname{Ker}\left(\right.\right.$ Aut $X^{\prime} \rightarrow$ Aut $\left.\left.H_{*}\left(X^{\prime}, Z\right)\right)\right) \subseteq \operatorname{Ker}\left(\right.$ Aut $X \rightarrow$ Aut $\left.H_{*}(X, Z)\right)$ is similar.

Proposition 4.4. Let $X$ and $X^{\prime}$ be as in 4.2. If Aut $X$ is finite then Aut $X$ is isomorphic to Aut $X^{\prime}$.

Proof. Let $h \in$ Aut $X$ and let $\varphi: X \rightarrow X_{0}$ be a rationalization. Since $X$ is an $H_{0}$-space and Aut $X$ is finite imply that Aut $X_{0}$ is abelian. For every prime $p$ and for every localization $\phi_{p}: X_{p} \rightarrow X_{0}$ $\phi_{p}\left(h_{p}\right)_{\varphi} \sim\left(h_{0}\right)_{\varphi} \phi_{p}$. Hence the proof is similar to the proof of Proposition 4.1.

Notations. Let $X$ be an $H_{0}$-space with either a finite number of homology groups or a finite number of homotopy groups, and let $\varphi: X \rightarrow K(X)(K(X))=K\left(Q H^{*}(X, Z) /\right.$ torsion $)$ be a rational equivalence. Denote by:

(a) $X(p, \varphi)$ the space which satisfies: There exists a factorization

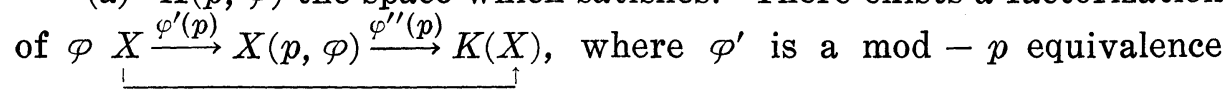
and $\varphi^{\prime \prime}$ is a $\bmod P-p$ equivalence. (Such a space exists by $[9,4.3 .1]$ ).

(b) $N(X)$ - the least integer which satisfies: For every $n>$ $N(Z)$ either $\pi_{n} X=0$ or $H_{n} X=0\left(\pi_{n}\right.$ - if $X$ has a finite number of homotopy groups, $H_{n}$ - if $X$ is finite dimensional).

(c) $t$ - the least integer divisible by

$$
\prod_{n \leqq N(X)}\left|\operatorname{torsion}\left(H^{n}(X, Z)\right)\right| \cdot \mid \operatorname{torsion}\left(\pi_{n}(\text { fiber } \varphi) \mid\right. \text {. }
$$


LEMma 4.5. Let $X$ and $\varphi$ be as in the notations. Then the map Aut $X(p, \varphi) \rightarrow$ Aut $X_{p}$ is monic.

Proof. Let $h \in \operatorname{Ker}\left(\operatorname{Aut} X(p, \varphi) \rightarrow \operatorname{Aut} X_{p}\right)$. Since $\operatorname{Ker}(\operatorname{Aut} X(p, \varphi) \rightarrow$ Aut $\left.X_{p}\right)$ contains $\operatorname{Ker}\left(\right.$ Aut $X(p, \varphi) \rightarrow$ Aut $\left.X_{0}\right)$ and $X(p, \varphi)$ is an $H_{0}$-space, $\varphi^{\prime \prime}(p) h \sim \varphi^{\prime \prime}(p)$, i.e., for every prime $p h$ is mod- $p$ homotopic to the identity, hence $h$ is homotopic to the identity [5, II 5.3].

Proposition 4.6. Let $X$ be an $H_{0}$-space either with a finite number of homology groups or with a finite number of homotopy groups. If $H^{*}(X, Z)$ is torsion free, then $\operatorname{Ker}\left(\right.$ Aut $X \rightarrow$ Aut $\left.X_{q}\right)(q \in P)$ is a direct product of finite $p$-groups, $p \neq q, p / t$.

Proof. Let $\phi: X \rightarrow K(X)$ be a rational equivalence which represents generators of $H^{*}(X, Z)$. By Lemma $4.5 \operatorname{Ker}\left(\right.$ Aut $X \rightarrow$ Aut $X_{p}$ ) is isomorphic to $\operatorname{Ker}($ Aut $X \rightarrow \operatorname{Aut} X(p, \varphi))$. Hence the fact that $X$ is the pullback of the maps $X(p, \varphi) \stackrel{p^{\prime \prime}(\varphi)}{\longrightarrow} K(X)(p / t)[9,4.7 .2]$ and that $\operatorname{Ker}($ Aut $X(p, \varphi) \rightarrow$ Aut $K(X))$ is a finite $p$-group [10, 2.9] implies the result.

Proposition 4.7. Let $X, Y$ be nilpotent spaces with finite number of homology groups and let $f: X \rightarrow Y$ be a fibration. Then for every $f^{\prime} \in G(f)\left(f^{\prime}: X^{\prime} \rightarrow Y^{\prime}\right)$ and for every prime $p, \operatorname{Ker}\left(\operatorname{Aut}(f) \rightarrow \operatorname{Aut}\left(f_{p}\right)\right)$ $i$ is isomorphic to $\operatorname{Ker}\left(\operatorname{Aut}(f) \rightarrow \operatorname{Aut}\left(f_{p}\right)\right)$ is isomorphic to $\operatorname{Ker}\left(\operatorname{Aut}\left(f^{\prime}\right) \rightarrow\right.$ $\left.\operatorname{Aut}\left(f_{p}^{\prime}\right)\right)$.

Proof. Let $\varphi: X \rightarrow X_{0}, \psi: Y \rightarrow Y_{0}, \varphi^{\prime}: X^{\prime} \rightarrow X_{0}$ and $\psi^{\prime}: Y^{\prime} \rightarrow Y_{0}$ be rationalizations. Assume that $f_{p}$ is the localization of $f$ with respect to $\varphi$ and $\psi$ and that $f_{p}^{\prime}$ is the localization of $f^{\prime}$ with respect to $\varphi^{\prime}$ and $\psi^{\prime}$. Since $f_{p}^{\prime}$ is homotopy equivalent to $f_{p}$ one can choose decompositions of $\varphi^{\prime}$ and $\psi^{\prime}$
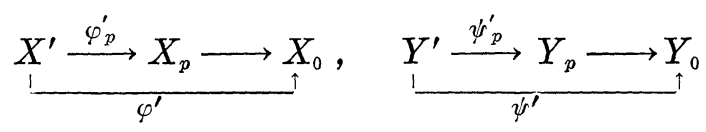

so that $f_{p} \varphi_{p}^{\prime} \sim \psi_{p}^{\prime} f^{\prime}[6,2.1 .2]$. Consequently, the considerations of the proof of Proposition 4.1 imply that for every pair $(h, k) \in$ Aut $f$ there exists a unique pair $\left(h^{\prime}, k^{\prime}\right) \in$ Aut $f^{\prime}$, which satisfies $\left(\left(h_{p}^{\prime}\right)_{\varphi^{\prime}},\left(k_{p}^{\prime}\right)_{\psi^{\prime}}\right)=$ $\left(\left(h_{p}\right)_{\varphi},\left(k_{p}\right)_{\psi}\right)$ for every prime $p$ and therefore $\operatorname{Ker}\left(\operatorname{Aut}(f) \rightarrow \operatorname{Aut}\left(f_{p}\right)\right)$ is isomorphic to $\operatorname{Ker}\left(\operatorname{Aut}\left(f^{\prime}\right) \rightarrow \operatorname{Aut}\left(f_{p}^{\prime}\right)\right)$.

Corollary 4.8. Let $X, Y$ be as in Proposition 4.2 and $f: X \rightarrow Y$ be a fibration. Then for every $f^{\prime} \in G(f) \operatorname{Ker}\left(\operatorname{Aut}(f) \rightarrow\right.$ Aut $\left.H^{*}(f, Q)\right)$ is isomorphic to $\operatorname{Ker}\left(\operatorname{Aut}\left(f^{\prime}\right) \rightarrow \operatorname{Aut} H^{*}\left(f^{\prime}, Q\right)\right)$. 
Corollary 4.9. Let $f$ and $f^{\prime}$ be as in Corollary 4.8. Then $\operatorname{Ker}\left(\operatorname{Auf}(f) \rightarrow\right.$ Aut $\left.H_{*}(f, Z)\right)$ is isomorphic to

$\operatorname{Ker}\left(\operatorname{Aut}\left(f^{\prime}\right) \longrightarrow \operatorname{Aut} H_{*}\left(f^{\prime}, Z\right)\right)$.

Proof. Similar to the proof of Corollary 4.3.

Proposition 4.10. Let $f$ and $f^{\prime}$ be as in Corollary 4.8. If Aut $(f)$ is finite, then $\operatorname{Aut}(f)$ is isomorphic to $\operatorname{Aut}\left(f^{\prime}\right)$.

Proof. Let $\varphi: X \rightarrow X_{0}$ and $\psi: Y \rightarrow Y_{0}$ be rationalizations. Since $f_{0}$ is homotopy equivalent to $f_{0}^{\prime}$ one can choose rationalization $\varphi^{\prime}: X^{\prime} \rightarrow$ $X_{0}$ and $\psi^{\prime}: Y^{\prime} \rightarrow Y_{0}$ so that $f_{0} \varphi^{\prime} \sim \psi^{\prime} f^{\prime}$. Hence the result follows from the fact that Aut $X_{0}$ and Aut $Y_{0}$ are abelian groups. (The proof is similar to the proof of Proposition 4.7).

\section{REFERENCES}

1. A. Borel, Linear Algebric Groups, Benjamin, New York, 1969.

2. C. R. Curjel, On the H-space structure of finite complexes, Comment. Math. Helvetici, 43 (1968), 1-17.

3. Sem. H. Cartan, ENS, 1959/60.

4. M. Daniel and J. R. Sunday, The self equivalences of an H-space, Pacific J. Math., 49 (1973), 507-517.

5. P. Hilton, G. Mislin and J. Roitberg, Localization in nilpotent groups and spaces, North Holland Math. Studies, 15 (1975).

6. S. Hurvitz, The genus of spaces and fibrations, Thesis, The Hebrew University.

7. J. P. Serre, Cohomology galosienne, Lecture Notes in Math., No. 5, Springer-Verlag, Berlin, (1965).

8. C. W. Wilkerson, Applications of minimal simplicial groups, Topology, 15 (1976), 111-130.

9. A. Zabrodsky, Hopf-Spaces, North Holland Math. Studies, 22 (1976).

10. - Endomorphisms in the homotopy category, mimeographed.

Received November 6, 1979.

BAR-ILAN UNIVERSITY

RAMAT-GAN, ISRAEL 


\section{PACIFIC JOURNAL OF MATHEMATICS}

\section{EDITORS}

DoNALD BABBITT (Managing Editor)

University of California

Los Angeles, CA 90024

Hugo Rossi

University of Utah

Salt Lake City, UT 84112

C. C. MOORE and ANDREw OGG

University of California

Berkeley, CA 94720
J. DugundJI

Department of Mathematics

University of Southern California

Los Angeles, CA 90007

R. FinN and J. Milgram

Stanford University

Stanford, CA 94305

ASSOCIATE EDITORS
R. ARENS
E. F. BECKENBACH
B. H. NeumanN
F. WOLF
K. YoshidA

\section{SUPPORTING INSTITUTIONS}

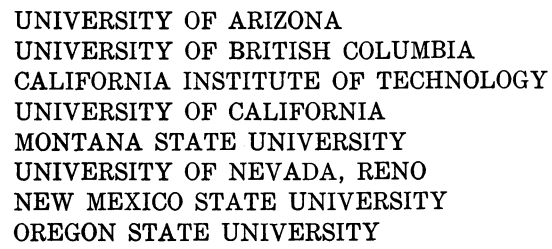

UNIVERSITY OF ARIZONA

UNIVERSITY OF BRITISH COLUMBIA

CALIFORNIA INSTITUTE OF TECHNOLOGY

UNIVERSITY OF CALIFORNIA

MONTANA STATE UNIVERSITY

UNIVERSITY OF NEVADA, RENO

NEW MEXICO STATE UNIVERSITY OREGON STATE UNIVERSITY

\author{
UNIVERSITY OF OREGON \\ UNIVERSITY OF SOUTHERN CALIFORNIA \\ STANFORD UNIVERSITY \\ UNIVERSITY OF HAWAII \\ UNIVERSITY OF TOKYO \\ UNIVERSITY OF UTAH \\ WASHINGTON STATE UNIVERSITY \\ UNIVERSITY OF WASHINGTON
}

The Supporting Institutions listed above contribute to the cost of publication of this Journal, but they are not owners or publishers and have no responsibility for its content or policies.

Mathematical papers intended for publication in the Pacific Journal of Mathematics should be in typed form or offset-reproduced, (not dittoed), double spaced with large margins. Please do not use built up fractions in the text of the manuscript. However, you may use them in the displayed equations. Underline Greek letters in red, German in green, and script in blue. The first paragraph or two must be capable of being used separately as a synopsis of the entire paper. Please propose a heading for the odd numbered pages of less than 35 characters. Manuscripts, in triplicate, may be sent to any one of the editors. Please classify according to the scheme of Math. Reviews, Index to Vol. 39. Supply name and address of author to whom proofs should be sent. All other communications should be addressed to the managing editor, or Elaine Barth, University of California, Los Angeles, California, 90024.

50 reprints to each author are provided free for each article, only if page charges have been substantially paid. Additional copies may be obtained at cost in multiples of 50 .

The Pacific Journal of Mathematics is issued monthly as of January 1966. Regular subscription rate: $\$ 102.00$ a year (6 Vols., 12 issues). Special rate: $\$ 51.00$ a year to individual members of supporting institutions.

Subscriptions, orders for numbers issued in the last three calendar years, and changes of address shoud be sent to Pacific Journal of Mathematics, P.O. Box 969, Carmel Valley, CA 93924, U.S.A. Old back numbers obtainable from Kraus Periodicals Co., Route 100, Millwood, NY 10546.

\footnotetext{
PUBLISHED BY PACIFIC JOURNAL OF MATHEMATICS, A NON-PROFIT CORPORATION

Printed at Kokusai Bunken Insatsusha (International Academic Printing Co., Ltd.). 8-8, 3-chome, Takadanobaba, Shinjuku-ku, Tokyo 160, Japan.
} 


\section{Pacific Journal of Mathematics}

\section{Vol. 96, No. 2 December, 1981}

Gerald A. Beer, A natural topology for upper semicontinuous functions and

a Baire category dual for convergence in measure $\ldots \ldots \ldots \ldots \ldots \ldots 251$

Georgia Benkart and J. Marshall Osborn, An investigation of real

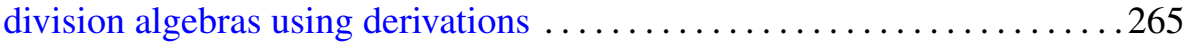

Donald Ian Cartwright and John R. McMullen, A structural criterion for the existence of infinite Sidon sets ........................ 301

Philip Hanlon, The fixed-point partition lattices $\ldots \ldots \ldots \ldots \ldots \ldots \ldots \ldots$

Eric Hayashi, The spectral density of a strongly mixing stationary Gaussian process

Chung-Wu Ho and Charles E. Morris, Jr., A graph-theoretic proof of

Sharkovsky's theorem on the periodic points of continuous functions . ...361

Sara Hurvitz, The automorphism groups of spaces and fibrations ....... 371

Atsushi Inoue, Schoichi Ota and Jun Tomiyama, Derivations of operator algebras into spaces of unbounded operators . .................. 389

Wolfgang B. Jurkat and Gary Sampson, On weak restricted estimates and endpoint problems for convolutions with oscillating kernels. I ........ 405

Georgios Koumoullis, Some topological properties of spaces of

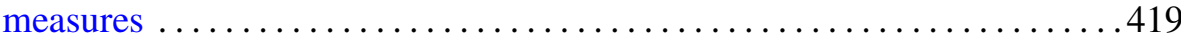

Wen Hsiung Lin, Algebraic Kahn-Priddy theorem ................. 435

Michael John McAsey, Invariant subspaces of nonselfadjoint crossed

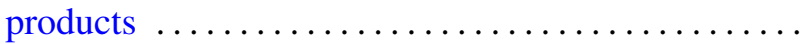

Justin Peters, Entropy of automorphisms on L.C.A. groups

Saburou Saitoh, A characterization of the adjoint $L$-kernel of Szegó type 